\title{
ACIDOSE TUBULAR RENAL DISTAL MANIFESTANDO-SE COM RABDOMIÓLISE
}

\author{
EDMAR ZANOTELI*, ACARY SOUZA BULLE DE OLIVEIRA*, CÉLIA HARUMI TENGAN*, \\ MARIA DA PENHA ANANIAS MORITA*, BENY SCHMIDT**, ALBERTO ALAIN GABBAI*
}

\begin{abstract}
RESUMO - A hipocalemia severa é causa incomum de rabdomiólise. Descreve-se o caso de uma paciente de 28 anos com acidose tubular renal distal, que desenvolveu hipocalemia severa com consequente rabdomílise. 0 estudo histológico do músculo evidenciou áreas focais de necrose muscular predominando em fibras do tipo II, com discreta reação macrofágica. A melhora clínica e laboratorial apresentada pela paciente ocorreu após a normalização do potássio sérico, tendo sido fundamental, neste caso, a correção da acidose metabólica.
\end{abstract}

PALAVRAS-CHAVE: hipocalemia, rabdomiólise, acidose tubular renal.

\section{Distal renal tubular acidosis manifesting with rhabdomyolysis}

SUMMARY - Severe hypokalemia is an uncommon cause of rhabdomyolysis. We describe a patient, 28-yearold woman, with distal renal tubular acidosis (DRTA) who developed severe hypokalemia and rhabdomyolysis. Muscle biopsy shows focal muscular necrosis mainly in type II muscle fibers and mild macrophagic reaction. After correcting the acidosis with oral administration of alkalinizing salts, clinical and laboratory improvement was seen. This clearly establish a causal realtionship between the positive acid balance, hypokalemia and the muscular manifestation in DRTA.

KEY WORDS: hypokalemia, rhabdomyolysis, renal tubular acidosis.

Rabdomiólise é a lesão da musculatura esquelética com perda da integridade da membrana celular e consequente extravazamento do sarcoplasma: creatinoquinase (CK), dehidrogenase láctica, aldolase, mioglobina e potássio - entre outros - para o plasma ${ }^{8}$. Suas causas podem ser classificadas em traumáticas e não-traumáticas, sendo elas um grupo extenso e diverso de afeç̧ões decorrentes de defeitos genéticos, alterações endocrinológicas, doenças imunologicas, infecções, drogas, toxinas, distúrbios metabólicos e hidroeletrolíticos ${ }^{8,15,19}$. A depleção de potássio é um dos distúrbios que pode levar à rabdomiólise $\mathrm{s}^{5,6,10,16} \mathrm{e}$ ocorre no organismo por baixa ingestão alimentar e por perdas deste íon pelo trato gastrointestinal ou renal.

Relatamos neste estudo o caso de uma paciente com acidose tubular renal distal (ATRD) que desenvolveu hipocalemia e consequente rabdomiólise, procurando ressaltar aspectos clínicos e fisiopatologicos desta associação.

*Disciplina de Neurologia, Departamento de Neurologia, Neurocirurgia e Neurologia Experimental, Escola Paulista de Medicina (EPM); **Departamento de Anatomia Patológica, EPM. Aceite: 13-junho-1994.

Dr. Acary S.B. Oliveira - Disciplina de Neurologia, Escola Paulista de Medicina - Rua Botucatu 740 - 04023 062 São Paulo SP - Brasil. 


\section{RELATO DO CASO}

EMJS, 28 anos, sexo feminino, parda, natural do Piauí e procedente de São Paulo, na $15^{\mathbf{a}}$ semana de gestação, procurou nosso Serviço com história de náuseas e vômitos há 15 dias acompanhados de fraqueza muscular inicialmente nos membros superiores e posteriormente nos membros inferiores, além de mialgia difusa. O quadro evolui com piora progressiva havendo incapacidade para deambulação. Negou uso de medicaçōes ou drogas anteriormente a este quadro ou episódios de trauma. Não apresentava antecedentes significaticos ou história familiar semelhante. Ao exame físico mostrou-se com hipotonia muscular, dor muscular difusa à palpação, hiporreflexia global, força muscular grau 3 proximal nos membros inferiores e superiores, grau 4 distal nos membros inferiores e superiores e na musculatura cervical. $O$ restante do exame clínico e neurológico foram normais. Os exames laboratoriais iniciais revelaram: creatirina $1,0 \mathrm{mg} / \mathrm{dL}(0,2-1,2 \mathrm{mg} / \mathrm{dL})$, s6dio $142 \mathrm{mEq} / \mathrm{L}$ (132-142 mEq/L), potássio 1,8 mEq/L (3,5-5,0 mEq/dL), glicemia $112 \mathrm{mg} / \mathrm{dL}$ (76-115 mg/dL), hemoglobina $10,6 \mathrm{~g} / \mathrm{dL}(12-16 \mathrm{~g} / \mathrm{dL})$, hematócrito $32 \%(37-47 \%)$, leucócitos $12700 \mathrm{~mm}^{3}$ (bastões $11 \%$, segmentados $68 \%$, eosinófilos $1 \%$, linfócitos $18 \%$, monócitos $2 \%$ ), transaminase glutâmica oxalacética 71 U/L (<15 U/L), CK $11.460 \mathrm{U} / \mathrm{L}$ (10-80 U/L), CK-mb $292 \mathrm{U} / \mathrm{L}$ (0-10 U/L), aldolase 5,5 U/L (<3,3 U/L).

A biópsia muscular, feita na vigência de fraqueza muscular, mostrou fibras necróticas em diversos estágios de degeneração além da presença de fibras atróficas isoladas (Fig 1 e Fig 2), predominantemente em fibras do tipo II.

A correção inicial do potássio ministrado na forma de cloreto de potássio $(60 \mathrm{mEg} /$ dia via oral, mais 100 $\mathrm{mEg} /$ dia via endovenosa) nos 2 primeiros dias da internação não foi acompanhada de normalização do seu nível sérico e nem de melhora na força muscular, apesar do desaparecimento dos vômitos. Os exames laboratoriais no segundo dia de internação revelaram: cretinina $0,8 \mathrm{mg} / \mathrm{dL}$, sódio $137 \mathrm{mEq} / \mathrm{L}$, potássio $2,2 \mathrm{mEq} / \mathrm{L}$, cloro 117 $\mathrm{mmol} / \mathrm{L}$ (96-105 mmol/L), magnésio 2,3 mg/dL (1,9-2,5 mg/dL), CK 4.500 U/L, CK-mb $156 \mathrm{U} / \mathrm{L}$; gasometria venosa: pH 7,23 (7,32-7,42), $\mathrm{HCO}^{3} 9,4 \mathrm{mmol} / \mathrm{L}(24-28 \mathrm{mmol} / \mathrm{L}), \mathrm{BE}-16,8 \mathrm{mmol} / \mathrm{L}(-2 \mathrm{a}+2 \mathrm{mmol} / \mathrm{L})$; urina tipo I: pH 8,0, leucócitos $6.000 / \mathrm{mL}$ (até $10.000 / \mathrm{mL}$ ), hemácias $1.000 / \mathrm{mL}$ (até $10.000 / \mathrm{mL}$ ); prova de acidificação urinária: pH 7,1 (6,0 a 6,7), acidez titulável $1,9 \mu \mathrm{Eq} / \mathrm{min}(6,4$ a $15,4 \mu \mathrm{Eq} / \mathrm{min})$, excreção de amônia $10 \mu \mathrm{Eq} / \mathrm{min}$ (18,3 a 38,3 $\mu$ microEq/min.). Clinicamente, a paciente mantinha o mesmo grau de fraqueza muscular da internação. A partir do segundo dia de internação foi mantida reposição de cloreto de potássio na dose diária de $150 \mathrm{mEq}$ endovenoso e inciou-se suplementação de bicarbonato de sódio via oral (120 mEq/dia). No quarto dia da internação houve normalização do nível sérico do potássio e da acidose metabólica havendo melhora progressiva do quadro clínico. A melhora completa da força muscular com normalização no nível sérico das enzimas musculares ocorreu em torno do $15^{\circ}$ dia de internação hospitalar.

\section{COMENTÁRIOS}

Acidose metabólica hipeclorêmica com hipocalemia, $\mathrm{pH}$ urinário persistentemente alcalino juntamente com baixa excreção de amônia na urina, encontrados neste caso, sugeriram o diagnóstico de ATRD $^{17}$. Este é um distúrbio caracterizado por incapacidade do rim em reduzir o $\mathrm{pH}$ urinário abaixo de 5,5 devido a defeito na secreção ou na manutenção do gradiente de hidrogênio na luz tubular. O sódio, a nível tubular distal, é reabsorvido em troca com o hidrogênio nas células principais, enquanto nas células intercaladas o hidrogênio é secretado eletrogenicamente. Nas situações em que a secreção de hidrogênio é inadequada ocorre aumento da atividade das células principais com perda predominante de potássio ${ }^{1,17}$.

A história clínica associada à elevação das enzimas musculares séricas juntamente com os achados na biopsia muscular sugeriam o diagnóstico de rabdomiólise. A evolução clínica favorável após a normalização do potássio sérico e a exclusão de outras causas de rabdomiólise nos levaram a acreditar que esta foi secundária à hipocalemia e possivelmente agravada pela acidose. Tem-se postulado um possível efeito tóxico direto da acidose no músculo esquelético, devido a alterações no sistema respiratório celular, em pacientes com acidose láctica e cetoacidose diabética ${ }^{20}$. Neste caso, porém, a correção da acidose foi fundamental no sentido de promover redução das perdas renais de potássio facilitando a correção do seu nível sérico.

A associação de hipocalemia decorrente de ATRD com rabdomiólise tem sido raramente relatada na literatura ${ }^{5,9}$, sendo a primeira descrição feita por Campion e col., em 1972 ${ }^{5}$. Smith e col., 


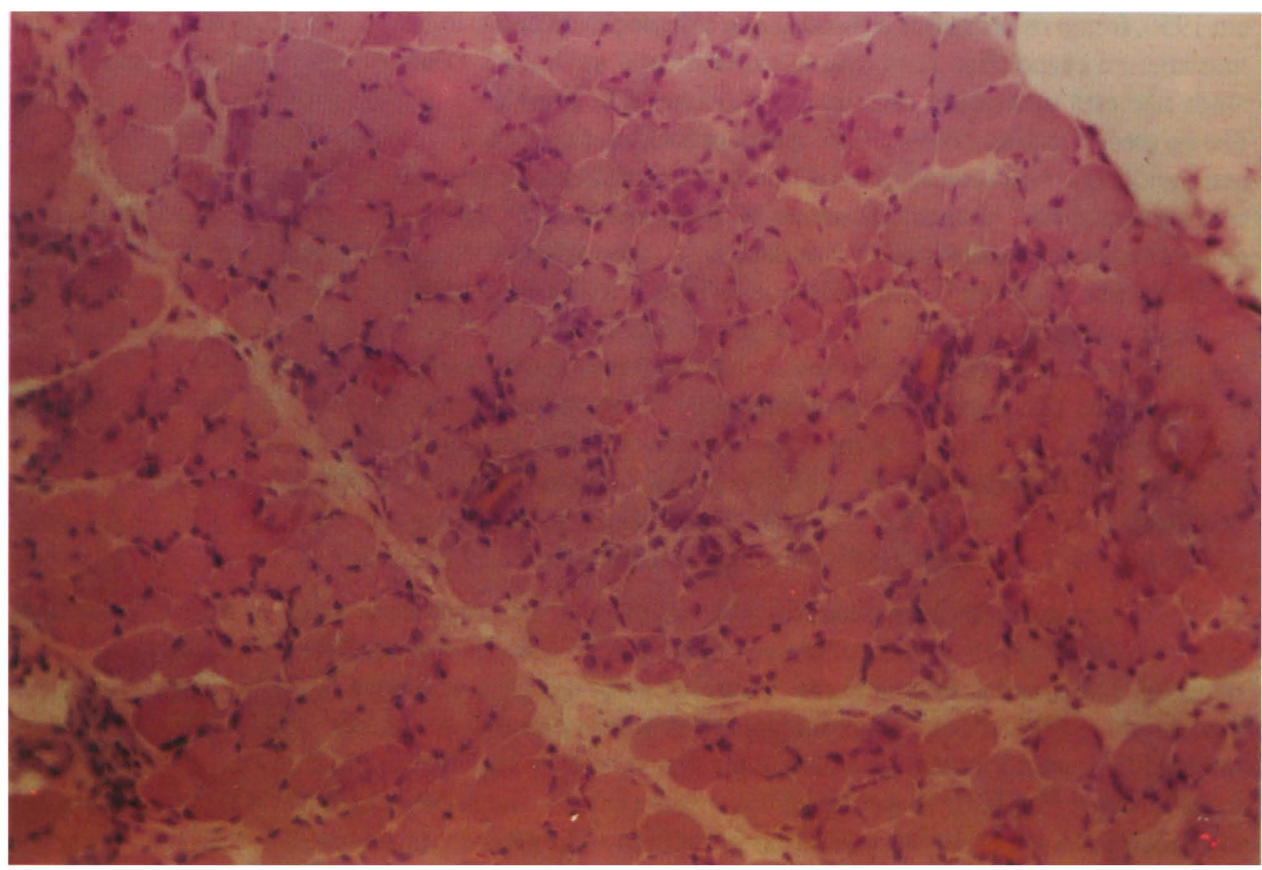

Fig 1. Presença de fibras necróticas e/ou degeneração com escassa reação macrofágica HE, $x 125$.

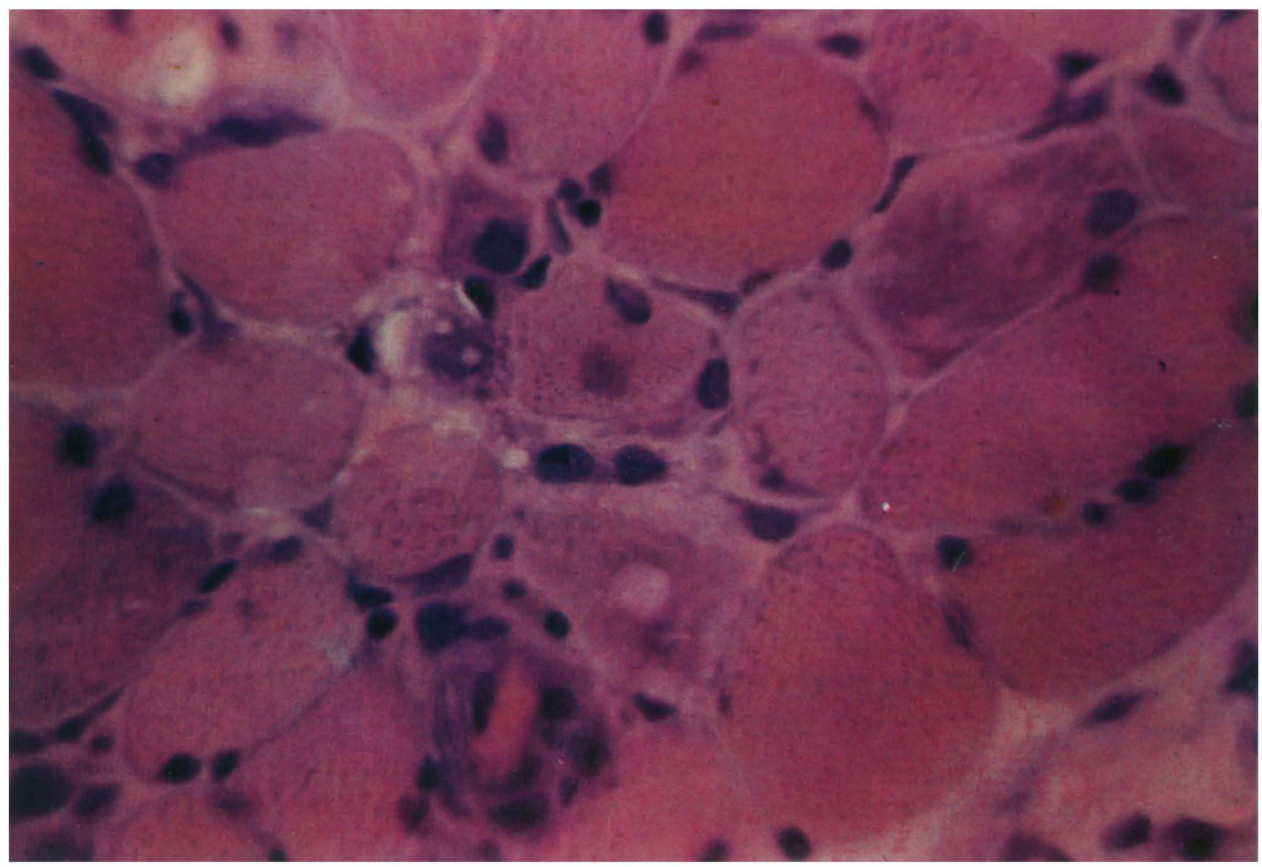

Fig 2. Detalhe das imagens de necrose eosinofilica com reação macrofágica HE, x 350 . 
em 1950, foram os primeiros a mostrar que a depleção de potássio leva a alterações degenerativas na musculatura esquelética num estudo experimental em cães ${ }^{23}$. O mecanismo pelo qual isso ocorre ainda não está totalmente esclarecido. Knochel e Schlein levantaram a hipótese de que isquemia fosse o mecanismo da rabdomiólise que ocorreu após expor cães depletados em potássio ao exercício extremo, já que nestas condições há liberação insuficiente de potássio da célula para o espaço extracelular, impedindo adequado aumento no fluxo sanguíneo para o tecido muscular ${ }^{16}$. A importância do potássio como mediador da vasodilatação no leito vascular do músculo após exercício havia sido mostrada por $\mathrm{Kjellmer}^{13}$. Um dado que reforça a teoria isquêmica de Knochel é que na miopatia isquêmica experimental são vistos na biopsia muscular achados semelhantes àqueles encontrados em pacientes com hipocalemia ${ }^{12}$. Quando há déficit prolongado de potássio ocorre perda da integridade da membrana plasmática, caindo o potencial de membrana a níveis muito baixos. Pode ocorrer, então, liberação da CK, como mostram estudos em cães depletados em potássio ${ }^{2,14}$. Além disso, o potássio tem importância em muitos processos metabólicos intracelulares, principalmente na glicólise $^{3.14}$. Assim, em certas situações, como no exercício físico, em que ocorre maior demanda energética e de oxigênio, a célula muscular se torna vulnerável à necrose.

Os achados na biopsia muscular da nossa paciente mostram áreas focais de necrose muscular, com fibras atroficas em vários estágios de degeneração e reação macrofágica. Não foi observado, no material examinado, degeneração vacuolar semelhante à encontrada na paralisia periódica hipocalêmica $(\mathrm{PPH})^{24}$, que esporadicamente é descrita na miopatia hipocalêmica ${ }^{5,6}$. A escassez de infiltrado inflamatório contrasta com a maioria dos relatos em que se mostra serem eles frequentes e que teriam a função de macrofagia ${ }^{6,7}$. A presença de atrofia seletiva para fibras do tipo II pode ser explicada pelo seu maior metabolismo glicolítico (não aeróbico), tornando-as mais vulneráveis à lesão ${ }^{7,21}$.

Um diagnóstico diferencial que deve ser lembrado é a PPH. Porém esta apresenta-se com crises periódicas de fraqueza muscular de início súbito e de pequena duração, com níveis de CK normais ou pouco elevados, não havendo depleção corporal total de potássio" ${ }^{11}$.

A evolução favorável apresentada pela nossa paciente assemelha-se à de outros relatos da literatura, os quais mostram que a correção da depleção de potássio promove normalização clínica com regeneração completa da fibra muscular ${ }^{6,10}$. $O$ tratamento da ATRD visa à correção da acidose metabólica crônica, podendo ser feito com administração de bicarbonato de sódio ou citrato de potássio em doses suficientes para correção do distúrbio metabólico ${ }^{17}$.

A complicação mais temível da rabdomiólise é a insuficiência renal aguda (IRA) que ocorre, mais provavelmente, devido a obstrução tubular renal pela precipitação da mioglobina ${ }^{4,15}$. A urina alcalina seria um fator protetor para esta complicação por reduzir a precipitação de proteínas nos túbulos renais ${ }^{4,15,18,19}$. Isto poderia explicar o motivo pelo qual pacientes com níveis tão altos de CK e urina alcalina, como no caso em questão, desenvolvem IRA numa frequência relativamente menor.

Singhal e col. estudaram 120 pacientes com potássio menor que $3,5 \mathrm{mmol} / \mathrm{L}$ de causas diversas, 38 dos quais tiveram evidência bioquímica de rabdomiólise, concluindo que em pacientes hipocalêmicos a rabdomiólise subclínica é condição comum, porém nem sempre notada ${ }^{22}$. É importante o reconhecimento precoce desta síndrome para promover adequado controle da doença de base e evitar complicações relacionadas à rabdomiólise, como: IRA e hipocalcemia ${ }^{8,19}$.

Agradecimentos - Agradecemos ao Prof. Dr. Nestor Schor, docente da Disciplina de Nefrologia da Escola Paulista de Medicina, pela prestigiosa colaboração na análise e correção deste estudo.

\section{REFERÊNCIAS}

1. Arruda JAL, Cowell G. Distal renal acidosis: molecular and clinical aspects. Hosp Pract 1994, 29: 75-88.

2. Bilbrey GL, Herbin L, Carter NW, Knochel JP. Skeletal muscle resting membrane potential potassium deficiency.

J Clin Invest 1973, 52: 3011-3018.

3. Blachley J, Long J, Knochel JP. The effect of potassium deficiency on resting muscle glycogen content and its response to exercise. Clin Res 1974, 21: 39A. 
4. Bywaters EGL, Stead JK. The production of renal failure following injection of solutions containing myohaemoglobin. Q J Exp Physiol 1944, 33: 53-70.

5. Campion DS, Arias JM, Carter NW. Rhadomyolysis and myoglobinuria: association with hypokalemia of renal tubular acidosis. JAMA 1972, 220: 967-969.

6. Comi G, Testa D, Cornelio F, Comola M, Canal N. Potassium depletion myopathy: a clinical and morphological study of six cases. Muscle \& Nerve 1985, 8: 17-21.

7. Corbett AJ, Pollock M. Experimental potassium depletion myopathy. J Neurol Sci 1981, 49: 193-206.

8. Gabow PA, Kaehny WD, Kelleher SP. The spectrum of rhabdomyolysis. Medicine 1982, 61: 141-152.

9. Hanip MR, Cheong IKS, Chin GL, Khalid BAK. Rhabdomyolysis associated with hypokalemic periodic paralysis of renal tubular acidosis. Singapore Med J 1990, 31: 159-161.

10. Heitzman EJ, Patterson JF, Stanley MM. Myoglobinuria and hypokalemia in regional enteritis. Arch Intern Med 1962, 110: 155-162.

11. Johnsen T. Familial periodic paralysis with hypokalaemia. Dan Med Bull 1981, 281: 1-22.

12. Kelts KA, Kaiser KK. Experimental ischemic myopathy: effects of aortic ligation and serotonin. $J$ Neurol Sci 1979, 40: 23-27.

13. Kjellmer I. The potassium ion as a vasodilator during muscular exercise. Acta Physiol Scand 1965, 63: 460-468.

14. Knochel JP. Etiologies and management of potassium deficiency. Hosp Pract 1987, 15: 153-162.

15. Knochel JP. Rhabdomyolysis and myoglobinuria. In Suki WN, Knoyan GE (eds). The kidney in systemic disease. Ed 2. New York: John Wiley \& Sons, 1981, p 263-284.

16. Knochel JP, Schlein EM. On the mechanism of rhabdomyolysis in potassium depletion. J Clin Invest 1972, 51: $1750-1758$.

17. Morris RC Jr, Ives HE. Inherited disordes of the renal tubule. In Brenner BM, Rector FC Jr (eds). The Kidney. Ed 4. Philadelphia: W.B. Saunders 1991, Vol II, p 1596-1656.

18. Peni GC, Gorini P. Uraemia in the rabbit after injection of crystalline myoglobin. Br J Exp Pathol 1952, 33: 440-444.

19. Penn AS. Myoglobinuria. In Engel AG, Banker BQ (eds). Myology. New York: McGraw-Hill, 1986, Vol II, p 1785-1805.

20. Rainey RL, Estes PW, Neeley CL, Amick LD. Myoglobinuria following diabetic acidosis with electromyographic evaluation. Arch Intern Med 1963, 111: 564-571.

21. Romanul FCA. Capillary supply and metabolism of muscle fibers. Arch Neurol 1965, 12: 497-509.

22. Singhal PC, Abramovici M, Venkatesan J, Mattana J. Hypokalemia and rhabdomyolysis. Miner Electrolyte Metab 1991, 17: 335-339.

23. Smith SG, Black-Schaffer B, Lasater TE. Potassium deficiency syndrome in the rat and dog. Arch Path 1950, 49: 185-199.

24. Tengan CH, Oliveira ASB, Morita MPA, Kiyomoto BH, Shmidt B, Gabbai AA. Paralisia periódica: estudo anátomo-patológico do músculo esquelético de 14 pacientes. Arq Neuropsiquiatr 1994, 52: 32-40. 